\title{
Psychiatrische Sprechstunde im Internet
}

\section{Psychiatric Consultation via Internet}

\section{Bibliografie}

DOI 10.1055/s-0029-1220458

Psychiat Prax 2009; 36:

308-310

(c) Georg Thieme Verlag KG

Stuttgart · New York .

ISSN 0303-4259

Korrespondenzadressen

Dr. med. Kai von Harbou

Bertha-v.-Suttner-Straße 8

14469 Potsdam

Kai@doctr.com

\section{Dr. Hans Kordy}

Forschungsstelle für Psychotherapie

Universitätsklinikum Heidelberg Bergheimer Straße 54 69115 Heidelberg

hans.kordy@med.uni-

heidelberg.de

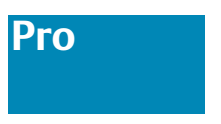

Digitale Medien haben mittlerweile in nahezu allen Bereichen des täglichen Lebens eine zentrale Rolle als Kommunikationskanal eingenommen. Auch für gesundheitsbezogene Themen wird das Internet auf Patientenseite in allen Altersgruppen in starkem und stetig steigendem Maße genutzt $[1,2]$.

Telemedizinische Verfahren, definiert als Erbringung medizinischer Leistungen ohne physischen Kontakt zwischen Patienten und Ärzten/nichtärztlichen Fachkräften bzw. zwischen Ärzten/ nichtärztlichen Fachkräften untereinander, werden seit Jahren in vielen Ländern wie z. B. Schweden, Norwegen oder den USA erfolgreich eingesetzt ([1-3], Gutachten im Auftrag des Ministerium für Gesundheit, Brandenburg, http://www. masgf.brandenburg.de/media/lbm1.a.1336.de/ telemedizin.pdf).

Zahlreiche internationale Studien belegen zudem den medizinischen, wie auch den ökonomischen Nutzen und berichten von einer hohen Akzeptanz unter Patienten und Leistungserbringern [5-10].

Dennoch hat sich die Nutzung von Telemedizin in der klinischen Betreuung von Patienten im deutschsprachigen Raum noch kaum etabliert. Dies erscheint um so verwunderlicher, wenn man berücksichtigt, dass durch bestehende Engpässe und v.a. regionale Disparitäten im Zugang zu medizinischen Leistungen bereits heute eine flächendeckende, bedarfsgerechte medizinische Versorgung in der Bundesrepublik vielerorts nicht mehr gewährleistet ist.

Aufgrund des zunehmenden Ärztemangels bei gleichzeitiger Alterung der Bevölkerung wird sich diese Entwicklung insbesondere in den strukturschwachen, ländlichen Regionen in $\mathrm{Zu}$ kunft weiter verschärfen. Der Einsatz von Informations- und Kommunikationstechnologie (IKT) ist daher unumgänglich, um auch in Zukunft eine bedarfsgerechte Gesundheitsversorgung zu ge- währleisten [Gutachten im Auftrag des Ministerium für Gesundheit, Brandenburg, s.o.].

Dass es zudem in Deutschland ohnehin ein erhebliches Potenzial für Qualitätssteigerungen in der Gesundheitsversorgung gibt, ist wohl unbestritten. Patienten, die über IKT mit ihren behandelnden Ärzten kommunizieren, schätzen dies als sehr effizient für die Behandlung ihrer Krankheit ein. Urness et al. untersuchten in einer Studie die Patientenzufriedenheit einerseits bei persönlicher und andererseits bei telepsychiatrischer Betreuung. Sie kamen zu dem Ergebnis, dass die per Videokonferenz betreuten Patienten nicht weniger zufrieden waren mit ihrer Behandlung als die persönlich Betreuten. Eine Reihe anderer internationaler Studien kommt zu ähnlichen Ergebnissen [4,8].

Die psychiatrische und psychosomatische Evaluation und Verlaufskontrolle von Patienten, bei der naturgemäß nicht die körperliche Untersuchung im Vordergrund steht, könnte bei der Nutzung von IKT eine Vorreiterrolle einnehmen, ist doch gerade hier der niederschwellige Zugang zum Therapeuten besonders wichtig.

Telepsychiatrie, in der Form einer interaktiven Videokonferenz, wie der Online-Sprechstunde, ist ein zeitsparendes, kostengünstiges und umweltfreundliches Mittel, um die Qualität und Quantität der Versorgung zu verbessern. Sie vermag durch höhere Patientenzufriedenheit und eine Verbesserung des Gesundheitszustandes langfristig zu einer Steigerung der Lebensqualität der Betroffenen beizutragen und die Übernahme von Eigenverantwortung bei den Patienten zu erhöhen. Auch telepsychiatrisch durchgeführte Konsile können eine zeitnahe und ökonomische Versorgung von Patienten in Krankenhäusern, Pflegeeinrichtungen und Praxen in den ländlichen Regionen ermöglichen, wo ein erschwerter Zugang zum Facharzt dies bislang häufig verhindert. 
Doch warum wird Telemedizin in Deutschland dann gegenwärtig höchstens projektartig praktiziert und steht nur in Einzelfällen an der Schwelle zur flächendeckenden Verbreitung?

Sicher hat das „digitale Desaster“ bei der missglückten Einführung der elektronischen Gesundheitskarte in Deutschland seinen Teil zu den Akzeptanzproblemen in der Ärzteschaft beigetragen. Als Begründung für die zögerliche Nutzung wird von Ärzteseite häufig aber auch ein „Fernbehandlungsverbot“ als rechtliche Hürde angeführt. Solch ein Verbot existiert jedoch nicht.

$\S 7$ Absatz 3 der Musterberufsordnung (MBO) bezieht sich nur auf eine ausschließliche Fernbehandlung und nicht auf die Behandlung oder Beratung via Telekommunikation innerhalb eines bestehenden Arzt-Patient-Verhältnisses. Laut Fachjuristen ist die Ausschließlichkeit sogar nicht auf einzelne Schritte oder einzelne Ärzte bezogen, sondern nur auf das gesamte Behandlungskonzept. Sie betrifft den einzelnen Arzt, dessen Behandlung oder Beratung durchaus auf die Telekommunikation beschränkt sein kann, also dann nicht, wenn der Patient in Bezug auf die Behandlung oder Erkrankung mit einem oder mehreren anderen Ärzten im persönlichen Arzt-Patienten-Kontakt steht [Ärzte Zeitung, 11.2.2009].

Inzwischen stehen ausgereifte Technologien für eine universelle Anwendbarkeit zur Verfügung, die in den Versorgungsprozess implementiert werden können. Auch der Gesetzgeber hat im Sozialgesetzbuch 5, §67 das Potenzial der elektronischen Kommunikation zur Verbesserung der Qualität und Wirtschaftlichkeit der Versorgung gewürdigt und verpflichtet Krankenkassen und Leistungserbringer sowie ihre Verbände zur finanziellen Unterstützung.

Zudem hat die europäische Kommission bereits 2008 von den Mitgliedsstaaten „die Schaffung von Vertrauen in die Dienste der Telemedizin und Aufbau von Akzeptanz, Schaffung von Rechtsklarheit und Klärung technischer Fragen und Erleichterung der Marktentwicklung der Telemedizin“ gefordert [KOM (2008) 689 endg.; Ratsdok. 15283/08].

Insgesamt ist ein grundsätzlicher Vorbehalt gegen die Telemedizin heute weder technologisch noch wissenschaftlich oder rechtlich begründbar. Es bleibt deshalb zu hoffen, dass auch und gerade in der Psychiatrie die Möglichkeiten der klinischen Telemedizin als Ergänzung und Erweiterung der herkömmlichen Versorgungsstrukturen in die Praxen Einzug hält.

Für nähere Informationen möchte ich auf meinen Blog unter www.doctr.com/blog hinweisen, wo wissenschaftliche Publikationen zu dem Thema zusammengetragen sind.

\section{Interessenkonflikte}

Der Autor ist Gründer und Geschäftsführer der Internet-Plattform doctr.com, die zurzeit eine Webanwendung zur Durchführung von Online-Sprechstunden entwickelt.



Seit der Vorstellung des Computerprogramms Eliza durch J. Weizenbaum in den 60ern fasziniert und schreckt der Gedanke, Computer(programme) könnten in die Rolle von Psychotherapeuten schlüpfen. Weizenbaum, damals einer der führenden Vertreter der künstlichen Intelligenz, hatte mit Eliza eine Software für die Verarbeitung natürlicher Sprache entwickelt und wollte diese an einem - öffentlichkeitswirksamen - Beispiel demonstrieren. Die Bereitschaft der Teilnehmer, den Computer als therapeutisches Gegenüber anzunehmen und intime Details preiszugeben, obwohl - oder gerade weil - sie wussten, dass sie mit einem Computer sprachen, überraschte ihn (und machte ihn zu einem ausdauernden Warner vor naiver Computergläubigkeit).

Auch heute greifen die Medien das Thema Mensch und Computer/Technik/ Automat, Tradition und Moderne dankbar auf; das schillernde Begriffspaar Technologie \& Ökonomie weckt gar das Interesse von Wirtschaftsjournalisten und lässt die Financial Times Deutschland verkünden „Arztvisiten per Internet entlasten Praxen und bieten Patienten zusätzlichen Service“, um dann zu klagen: „Die Kassen zahlen aber nicht.“ (FTD.de, 7.4.2009).

Ist es wirklich Zeit für die Psychiatrische Sprechstunde im Internet? Die Einführung von Neuem nur um des Neuen willen, bringt die Gesundheitsversorgung nicht weiter. Auch Not allein macht nicht aus jeder Innovation eine Tugend. Es geht nicht darum:

- die Welt mit technologischen Entwicklungen zu beglücken, die niemand braucht oder will. „It's People, Process and Technology - in That Order" mahnen die Autoren einer kürzlich publizierten internationalen Erhebung. Wie schnell auch technisch gute Entwicklungen zu Ladenhütern werden, zeigt sich an Beispielen wie EMMA (Engaging Media for Mental Health Application) oder SALUT (Intelligent Environment for Diagnostic, Treatment and Prevention of Eating Disorders). Diese von der EU großzügig geförderten Projekte suchen seit einigen Jahren vergeblich ihren Markt: IST-2001-39192; http://cordis.europa.eu/fetch?ACTION=D\&CALLER=PROJ_ IST\&RCN=64697.

- Psychotherapie neu zu erfinden, auch wenn manche Protagonisten gerne von Online-, Internet- oder computerisierter Psychotherapie sprechen und damit den Eindruck erwecken, durch den Einsatz dieser „Neuen“ Medien entstünden neue Wirkmechanismen. Die Frage, ob der Einsatz von IKT die psychosoziale Gesundheitsversorgung verbessert, ist ja nicht klüger als die Frage, ob der Einsatz von Medikamenten oder Psychotherapie dies tue. Eine wissenschaftlich fundierte Gesundheitsversorgung verlangt dagegen eine Klärung, was der Einsatz von IKT für wen unter welchen Bedingungen bringt.

- etablierte Versorgungsstrukturen aufzubrechen; auch wenn es manchmal den Anschein hat, dass Regeln wie das Fernbehandlungsverbot benutzt werden, um Veränderungen im Markt für Versorgung und Ausbildung zu verhindern, ist doch ihr vordringlicher Zweck, die Sicherheit der Patienten zu gewährleisten. Daher geht es darum zu klären, für wen der Einsatz von IKT unter welchen Umständen vorteilhaft oder schädlich ist, wie dieser Einsatz zu gestalten ist, damit die Vorteile möglichst groß und die Risiken möglichst klein werden. Dass dabei liebgewonnene Gewissheiten, wie etwa die Bedeutung der persönlichen Begegnung und Beziehung für die Psychotherapie (Hessische Psychotherapeutenkammer 2005) oder der nonverbalen Kommunikation relativiert werden, ist sicher ein interessanter wissenschaftlicher Nebeneffekt.

Innovationen wecken Erwartungen und Ängste - sowohl bei potenziellen Nutzern als auch bei potenziellen Anbietern. Die Erwartungen sind nicht unbegründet. Eine wachsende Zahl von Studien berichtet positive Ergebnisse [11]. Offensichtlich finden Menschen wirksame psychosoziale Unterstützung mithilfe von IKT. Allerdings dämpfen das „law of attrition“ [12] bzw. Dropout-Raten bis zu 80\% die Euphorie der Anhänger IKT-gestützter psychosozialer Dienste und lenken die Aufmerksamkeit auf die Gestaltung geeigneter Rahmenbedingungen (z.B. www.CHI 2008.org). 
Gegen die Ängste hilft ein umsichtiges Vorgehen. Schaut man beispielsweise nach Großbritannien [13]. Dort gibt es traditionell eine Versorgungsnot und einen hochentwickelten Pragmatismus. Seit 2006 erlaubt das National Institute for Health and Clinical Excellence (NICE) den Einsatz von zwei computerisierten Varianten der Kognitiven Verhaltenstherapie, „fear fighter“ für die Behandlung von Phobien und Ängsten und „Beat the Blues“ für die Behandlung von Depressionen. Die Zulassung erfolgt allerdings unter klaren Bedingungen: Beide computerisierten Verfahren werden im Rahmen einer „gestuften Versorgung“ nur bei „milder“ Erkrankung und in der Verantwortung spezialisierter Teams der Primärversorgung als Option zugelassen (z.B. http://guidance.nice.org.uk/CG23). Und es sind ausschließlich diese beiden, da nur für sie ausreichende wissenschaftliche Wirksamkeitsnachweise vorliegen.

Zurück nach Deutschland. Auch hier wächst die Evidenz für den Nutzen von IKT-gestützten spezifischen Angeboten für die Gesundheitsversorgung (z.B. [11]). Das ist jedoch kein Freifahrtschein für Trittbrettfahrer. Einige dieser Anwendungen, insbesondere in der sektorenübergreifenden Vorsorge („Frühintervention“) und Nachsorge nach stationärer Behandlung, haben sich wissenschaftlich so weit bewährt, dass die Übertragung in die Regelversorgung versucht werden kann. Die psychiatrische Sprechstunde ist bisher nicht dabei - und nicht von ungefähr: wissenschaftliche Untersuchungen sind bisher Fehlanzeige.

Dabei bieten sich durchaus Chancen (z. B. [14]). Der Patient kann zu Hause abgeholt und nach Hause begleitet werden. Unkenntnis und Stigmatisierung erschweren nach wie vor den Zugang zur psychiatrischen Sprechstunde und erhöhen das Risiko für Unter- und Fehlversorgung (z.B. auch durch naive Nutzung von Angeboten im Internet). Über das Internet ließen sich Orientierungshilfen geben und eine Begleitung in die psychiatrische Sprechstunde unaufwendig organisieren. Durch ein IKT-gestütztes Ergebnismonitoring ließen sich individueller Bedarf des Patienten und Umfang und Dauer der Therapie aufeinander abstimmen und damit ein Schritt zur Personalisierung der Versorgung machen; supportives Monitoring und Betreuung über Internet oder Mobiltelefon ermöglichen neue Strategien zur Behandlung bei chronischen Krankheitsverläufen. Das sind - nicht unrealistische - Möglichkeiten, aber für ihre verantwortungsvolle Nutzung stehen zunächst die wissenschaftlichen Hausaufgaben an.
Mit „Persuasion and Healing“ beschreibt Frank [15] in einem der Klassiker der Psychotherapiegeschichte einen zentralen Wirkmechanismus. Moderne IKT hat für manchen Patienten eine große Überzeugungskraft, die von einem geschickten Heiler zu seinem Wohle genutzt werden kann - es ist die Verantwortung der „Heiler“ in der Gesundheitsversorgung, die Chancen zu maximieren und die Risiken zu minimieren. Eine sorgfältige Abgrenzung des Indikationsbereiches und wissenschaftlich begleitete Modellversuche zur Testung und Erweiterung seiner Grenzen helfen Kurs zu halten auf dem schmalen Grat zwischen Mut und Übermut.

\section{Literatur}

1 Kummervold PE, Chronaki CE, Lausen B et al. eHealth Trends in Europe 2005-2007: A Population-Based Survey. J Med Internet Res 2008; 10 (4): e42

2 Baker L, Wagner TH, Singer S et al. Use of the Internet and E-mail for Health Care Information: Results From a National Survey. JAMA 2003; 289 (18): 2400-2406

3 Perednia DA, Allen A. Telemedicine technology and clinical applications. JAMA 1995; 273 (6): 483-488

4 Urness $D$ et al. Client acceptability and quality of life-telepsychiatry compared to in-person consultation. J Telemed Telecare 2009; 12: 251-254

5 Monnier J, Knapp R, Frueh B. Recent Advances in Telepsychiatry: An Updated Review. Psychiatr Serv Dec 2003; 54, 12: 1604-1609

6 Shore JH, Hilty DM, Yellowlees P. Emergency management guidelines for telepsychiatry. Gen Hosp Psychiatry 2007; 29: 199-206

7 Williams J, Ellis A, Middleton A et al. Primary care patients in psychiatric clinical trials: a pilot study using videoconferencing. Ann Gen Psychiatry 2007; 6: 24

8 O'Reilly R, Bishop J, Maddox $K$ et al. Is Telepsychiatry Equivalent to Face-to-Face Psychiatry? Results From a Randomized Controlled Equivalence Trial. Psychiatr Serv 2007; 58, 6: 836-843

9 Sulzberger $H$, Bullinger A, de las Cuevas $C$ et al. Die Psychiatrie im Internet und das Internet in der Psychiatrie. Neuropsychiatrie; Band 20, Nr. $3 / 2006$

10 Baker L, Rideout J, Gertler P et al. Effect of an Internet-Based System for Doctor-Patient Communication on Health Care Spending. J Am Med Inform Assoc 2005; 12: 530-536

11 Bauer S, Kordy H Hrsg. E-Mental-Health - Neue Medien in der psychosozialen Versorgung. Heidelberg: Springer, 2008

12 Eysenbach $G$. The law of attrition. J Med Internet Res 2005; 7: e11

13 Marks IM, Cavanagh K, Gega L. Computer-aided psychotherapy: revolution or bubble? Br J Psychiatry 2007; 191: 471-473

14 Hohagen F. Internetgestützte Therapieprogramme: Gefahr für die traditionelle Psychotherapie? Verhaltenstherapie 2009; 19: 4-5

15 Frank J. Persuasion and Healing (2nd ed). Baltimore: Johns Hopkins University Press, 1971 